\title{
EFFECTS OF SANDBLASTING CONDITIONS IN PREPARATION OF BIOACTIVE STAINLESS STEELS BY THE FUNCTION OF APATITE NUCLEI
}

\author{
Takeshi Yabutsuka $^{1 *}$, Ryoki Karashima $^{1}$, Shigeomi Takai ${ }^{1}$, Takeshi Yao ${ }^{2,3}$ \\ (*Corresponding author: yabutsuka@energy.kyoto-u.ac.jp) \\ ${ }^{1}$ Graduate School of Energy Science, Kyoto University, Kyoto 606-8501 Japan \\ ${ }^{2}$ National Institute of Technology, Kagawa College, Takamatsu, Kagawa 761-8058 Japan \\ ${ }^{3}$ Institute of Advanced Energy, Kyoto University, Uji, Kyoto 611-0011 Japan
}

Keywords: Apatite-forming ability, Apatite nuclei, Sandblasting process, Stainless steels, Surface roughness

\begin{abstract}
We formed many micropores on the surfaces of stainless steel (SUS) substrates by sandblasting method using alumina particles with $14 \mu \mathrm{m}$ or $3 \mu \mathrm{m}$ for average particle size and apatite nucleus (AN) treatment was operated. By these treatments, we provided bioactivity to the SUS substrates. We evaluated apatite-forming ability of the SUS substrate by soaking in a simulated body fluid. Apatite formation was induced on the surface of the substrate within 1 day. High adhesive strength of apatite layer was achieved by a mechanical interlocking effect between the apatite layer and the substrate. The adhesive strength was related to the size of the grinding particles in the sandblasting process.
\end{abstract}

(Received January 4, 2016; Accepted March 19, 2016)

\section{INTRODUCTION}

When the $\mathrm{pH}$ or the temperature of a simulated body fluid (SBF) with inorganic ion concentrations nearly equal to those of human blood plasma $^{1-4}$ is raised, fine particles of calcium phosphate are precipitated in the fluid. Recently, we found that the fine particles actively induce apatite formation and referred this fine particle as an apatite nucleus (AN) 5,6 .

In a series of our previous studies, we formed micropores on metalic materials and polymeric materials by sandblasting method and precipitated $\mathrm{AN}$ in the pores of each substrate and provided high bioactivity to these materials ${ }^{7-11}$. Those materials showed high adhesive strength between the bone-like apatite layer formed in SBF and each substrate.

Stainless steel (SUS) is one of the most traditional metalic biomaterials with high mechanical strength. From these properties, SUS has been already used as orthopedic implants such as artificial hip joint. However, SUS has no bioactivity. If bioactivity is provided to SUS, range of its clinical or dental application is largely extended.

In this study, we formed micropores on the surfaces of SUS substrates by the sandblasting method using two kinds of grinding particles and precipitated apatite nuclei in the pores of the substrate. We evaluated apatite-forming ability of the treated substrates, measured adhesive strength of apatite layer formed in SBF, and then investigated the relationship between the adhesive strength and the size of the grinding particles in the sandblasting process.

\section{MATERIALS AND METHODS}

1) Sandblasting process for micropores formation

We used commercially SUS plates (JIS SUS316L, Nisshin Steel, Japan) with $15 \times 10 \times 2$ $\mathrm{mm}^{3}$ in size as substrates. We treated the SUS substrates by sandblasting processes (PNEUMA-BLASTER ${ }^{\circledR}$ SFC-2, Fuji Manufacturing, Japan) at $0.85 \mathrm{MPa}$ of discharge pressure using alumina grinding particles with $14 \mu \mathrm{m}$ (JIS \#800) or $3 \mu \mathrm{m}$ (JIS \#4000) for average particle size. We washed the substrates in aceton, ethanol and distilled water by using an ultrasonic cleaner. We observed the surface morphologies of the substrates by field emission scanning electron microscopy (SEM; SU6600, Hitachi High-Technologies, Japan). We analyzed the surface roughness for each sandblasting condition by JIS B601:2001 (ISO 4287:1997) by using laser microscopy (VK-9700, KEYENCE, Japan). 
2) $\mathrm{AN}$ treatment for providing bioactivity to the substrate

We prepared SBF by dissolving reagent-grade $\mathrm{NaCl}, \mathrm{NaHCO}_{3}, \mathrm{KCl}, \mathrm{K}_{2} \mathrm{HPO}_{4} \cdot 3 \mathrm{H}_{2} \mathrm{O}, \mathrm{MgCl}_{2} \cdot 6 \mathrm{H}_{2} \mathrm{O}$, $\mathrm{CaCl}_{2}$, and $\mathrm{Na}_{2} \mathrm{SO}_{4}$ in an ultrapure water with the composition as shown in TABLE 1 . We subsequently raised the $\mathrm{pH}$ value to 8.40 by using tris(hydroxymethyl)aminomethane at $25.0{ }^{\circ} \mathrm{C}$. We soaked the SUS substrates in the solution and pressed the solution by cold isostatic pressing machine (CIP-SI, Kobe Steel, Japan) in $392 \mathrm{MPa}$ for $60 \mathrm{~min}$ to make the solution penetrate in the micropores. In order to precipitate $\mathrm{AN}$ in the pores of the specimens, we heated the substrates by using electromagnetic induction at $2.5 \mathrm{~kW}$ for 3 hours while soaking in the solution. We washed the substrates gently and air-dried. We denote these treatments as 'AN treatment' here after.

TABLE 1. Ion concentrations of human blood plasma and simulated body fluid (SBF)

\begin{tabular}{crr}
\hline \hline \multirow{2}{*}{ Ion } & \multicolumn{2}{c}{ Concentration $/ \mathrm{mmol} \cdot \mathrm{dm}^{-3}$} \\
\cline { 2 - 3 } & Blood plasma & \multicolumn{1}{c}{$\mathrm{SBF}$} \\
\hline $\mathrm{Na}^{+}$ & 142.0 & 142.0 \\
$\mathrm{~K}^{+}$ & 5.0 & 5.0 \\
$\mathrm{Mg}^{2+}$ & 1.5 & 1.5 \\
$\mathrm{Ca}^{2+}$ & 2.5 & 2.5 \\
$\mathrm{Cl}^{-}$ & 103.0 & 147.8 \\
$\mathrm{HCO}_{3}{ }^{-}$ & 27.0 & 4.2 \\
$\mathrm{HPO}_{4}{ }^{2-}$ & 1.0 & 1.0 \\
$\mathrm{SO}_{4}{ }^{2-}$ & 0.5 & 0.5 \\
$\mathrm{Others}$ & 23.0 & 0.0 \\
\hline $\mathrm{pH}$ & $7.25-7.40$ & 7.40 \\
\hline
\end{tabular}

\section{3) Evaluation of apatite-forming ability}

It was reported that the SBF test is a suitable in vitro method for predicting bioactivity of implant materials in vivo ${ }^{2-4,12}$. In this study, we evaluated an apatite-forming ability of the substrates for each sandblasting condition by soaking the substrates in SBF at $\mathrm{pH} 7.40,36.5^{\circ} \mathrm{C}$, based on the method of ISO/FDIS $23317^{3}$. After the soaking in SBF, we washed the substrates in distilled water and air-dried. We analyzed the apatite formation on the surface of the substrates by thin film X-ray diffraction (XRD; Rint 2500 , Rigaku, Japan) by using $\mathrm{Cu}-\mathrm{K} \alpha$ radiation, SEM and energy dispersive X-ray analysis (EDX; XFlash $^{\circledR}$ 5010, Bruker, Germany).

4) Measurement of adhesive strength of the apatite layer formed in SBF

We measured the adhesive strength between each substrate and the apatite layer formed by soaking in SBF for 14 days by a modified ASTM C-633 method $^{13,14}$. We attached both sides of each substrate to stainless steel jigs $\left(10 \times 10 \mathrm{~mm}^{2}\right)$ by Araldite ${ }^{\circledR}$ glue and applied a tensile load at $1 \mathrm{~mm} \cdot \mathrm{min}^{-1}$ of a crosshead speed until fracture occurred with universal testing machine (Model AGS-H Autogragh, Shimadzu).

\section{RESULTS AND DISCUSSION}

FIGURE 1 shows the SEM picture of the surface of the untreated SUS316L substrate. The grain boundaries were observed on the substrate.

FIGURE 2 and FIGURE 3 show the SEM pictures of the surface of the SUS316L substrates after sandblasting process using the $14 \mu \mathrm{m}$ or the 3 $\mu \mathrm{m}$ alumina grinding particles. Both substrates possessed the surface with complicated structures formed by the sandblasting process. However, the difference between each size of grinding particles was not clearly observed by SEM.

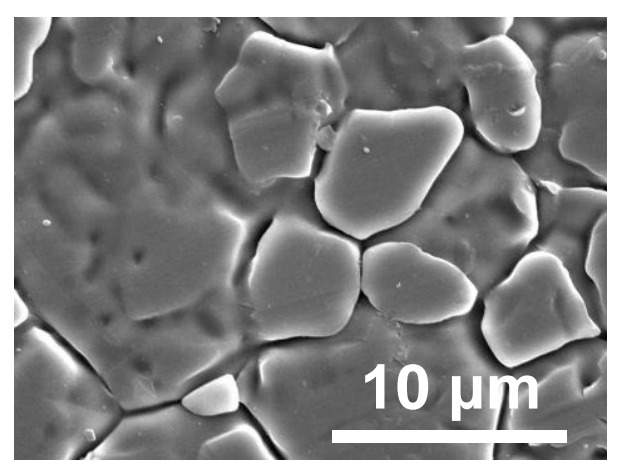

FIGURE 1. SEM picture of the surface of the untreated SUS316L substrate.

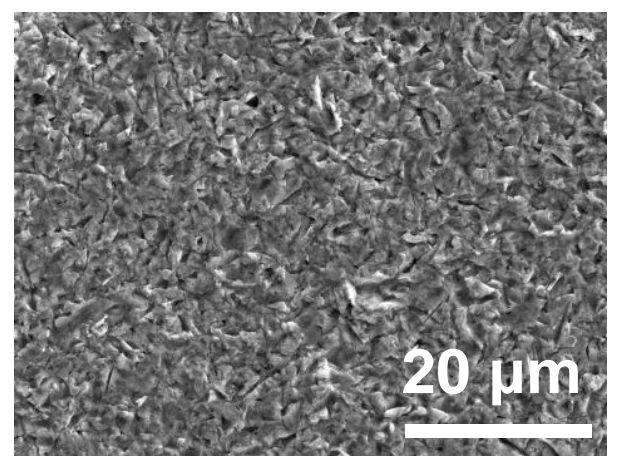

FIGURE 2. SEM picture of the surface of the SUS316L substrate after the sandblasting process using the $14 \mu \mathrm{m}$ grinding particles. 
FIGURE 4 and FIGURE 5 show the average surface roughness of the SUS316L substrates after the sandblasting process using the $14 \mu \mathrm{m}$ or the $3 \mu \mathrm{m}$ alumina grinding particles. In the case of the $14 \mu \mathrm{m}$ grinding particles, $\mathrm{R}_{\mathrm{a}}$ and $\mathrm{R}_{\mathrm{z}}$ were $0.47 \pm 0.04 \mu \mathrm{m}$ and $6.67 \pm 0.38 \mu \mathrm{m}$, respectively. In the case of the 3 $\mu \mathrm{m}$ grinding particles, $\mathrm{R}_{\mathrm{a}}$ and $\mathrm{R}_{\mathrm{z}}$ were $0.95 \pm 0.04 \mu \mathrm{m}$ and $8.55 \pm 0.47 \mu \mathrm{m}$, respectively. Both $R_{a}$ and $R_{z}$ of the case of the $3 \mu \mathrm{m}$ grinding particles were larger than that of the $14 \mu \mathrm{m}$. This result suggested that smaller alumina grinding particles are available to increase the surface roughness of the SUS316L substrate.

FIGURE 6 and FIGURE 7 shows the XRD patterns of the surface of the SUS316L substrate after the sandblasting process using the $14 \mu \mathrm{m}$ or the $3 \mu \mathrm{m}$ grinding particles, the AN treatment, and then soaking in SBF for various periods. For both conditions, diffraction peaks of apatite were clearly observed after soaking in SBF for 1 day, 3 days and 7 days. This means that high apatite-forming ability was achieved because the apatite formation was induced within 1 day.

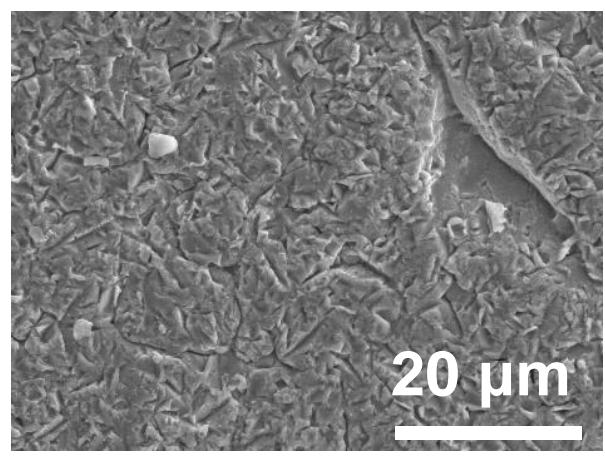

FIGURE 3. SEM picture of the surface of the SUS316L substrate after the sandblasting process using the $3 \mu \mathrm{m}$ grinding particles.

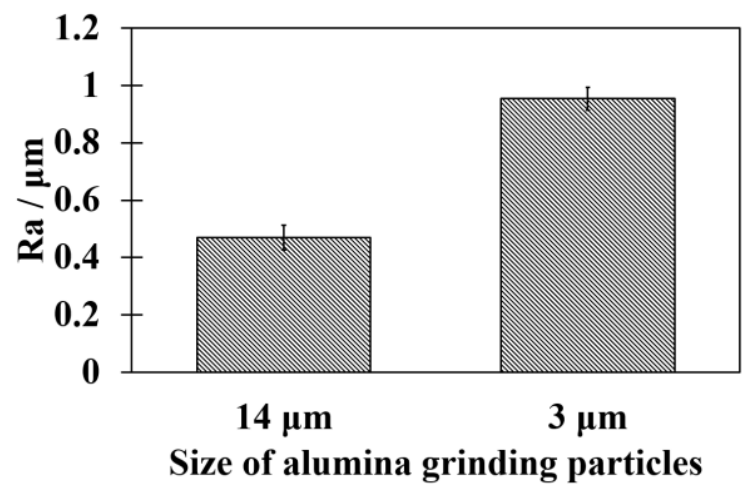

FIGURE 4. Average surface roughness $\left(R_{a}\right)$ of the SUS316L substrates after the sandblasting process using the $14 \mu \mathrm{m}$ or the $3 \mu \mathrm{m}$ alumina grinding particles.

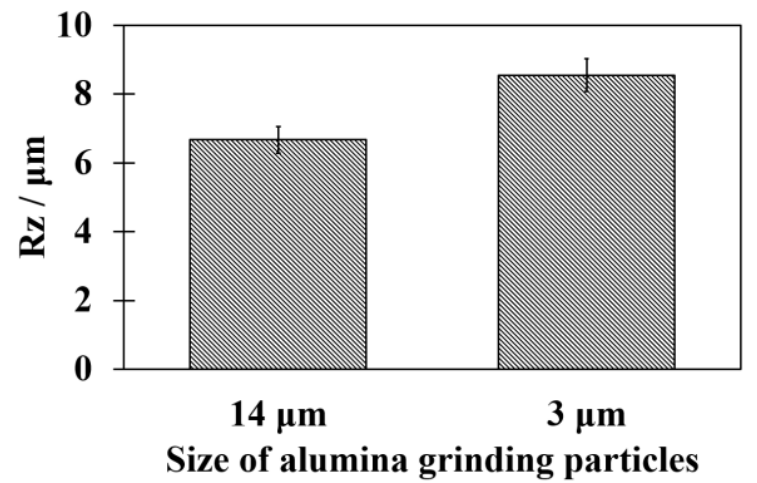

FIGURE 5. Average surface roughness $\left(R_{z}\right)$ of the SUS316L substrates after the sandblasting process using the $14 \mu \mathrm{m}$ or the $3 \mu \mathrm{m}$ alumina grinding particles.

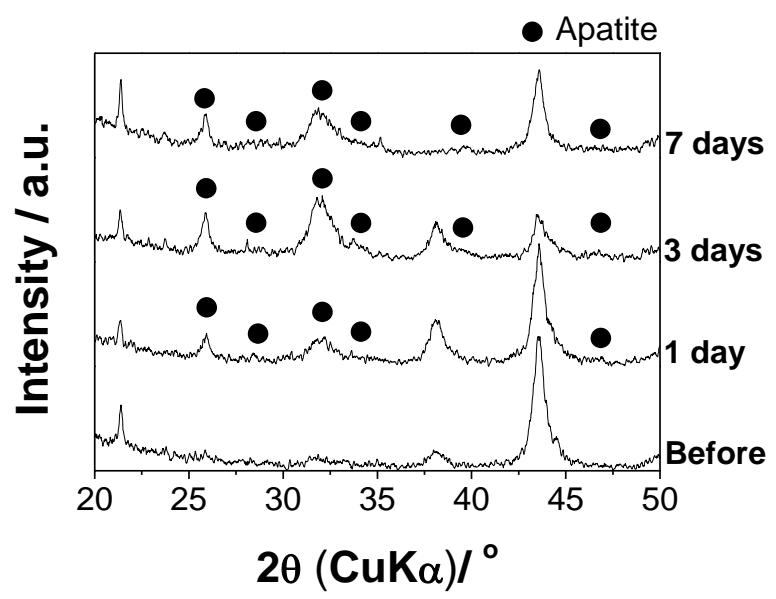

FIGURE 6. XRD patterns of the surface of the SUS316L substrate after the sandblasting process using the $14 \mu \mathrm{m}$ grinding particles, the AN treatment, and then soaking in SBF for various periods.

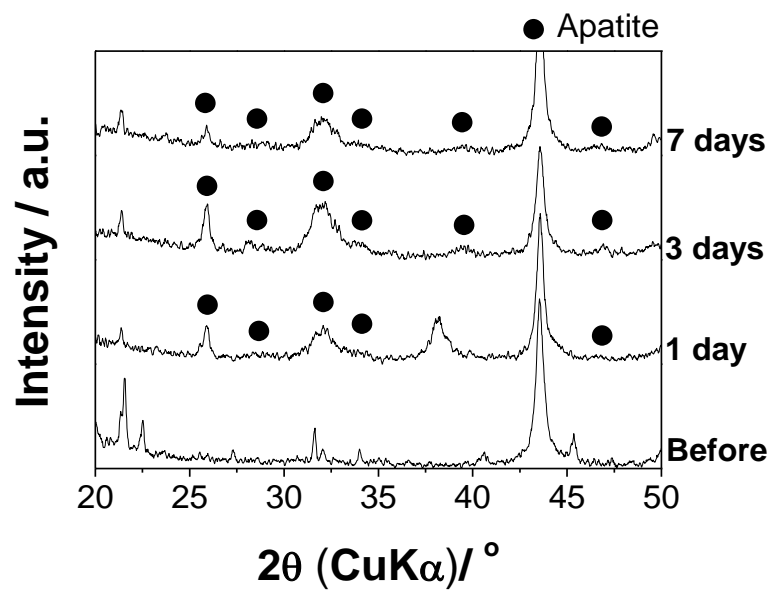

FIGURE 7. XRD patterns of the surface of the SUS316L substrate after the sandblasting process using the $3 \mu \mathrm{m}$ grinding particles, the AN treatment, and then soaking in SBF for various periods. 
FIGURE 8, FIGURE 9, FIGURE 10 and FIGURE 11 show the SEM pictures and the EDX results of the surfaces of the SUS316L substrates after the sandblasting process using the $14 \mu \mathrm{m}$ or the $3 \mu \mathrm{m}$ grinding particles, the AN treatment, and then soaking in SBF for 1 day. In each SEM picture, it was observed that the whole surface was covered with flake-like crystallites, which characterize bone-like apatite, for both sandblasting conditions. In each EDX result, the peaks of phosphorous and calcium were observed for both sandblasting condition. From these results, it is indicated that apatite formation was induced within 1 day and high apatite-forming ability was provided to the SUS by the AN treatment regardless of the sandblasting conditions.

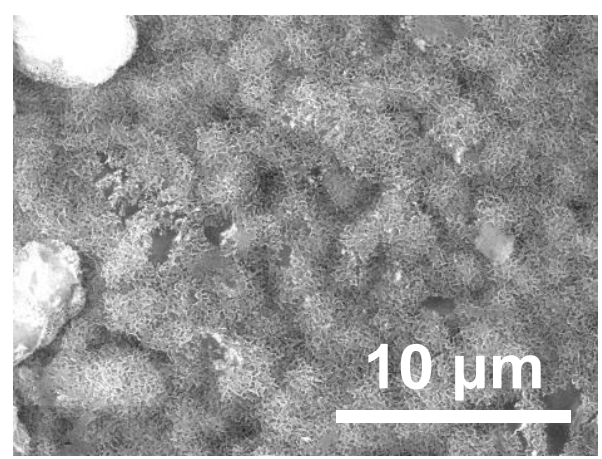

FIGURE 8. SEM picture of the surface of the SUS316L substrate after the sandblasting process using the $14 \mu \mathrm{m}$ grinding particles, the AN treatment, and then soaking in SBF for 1 day.

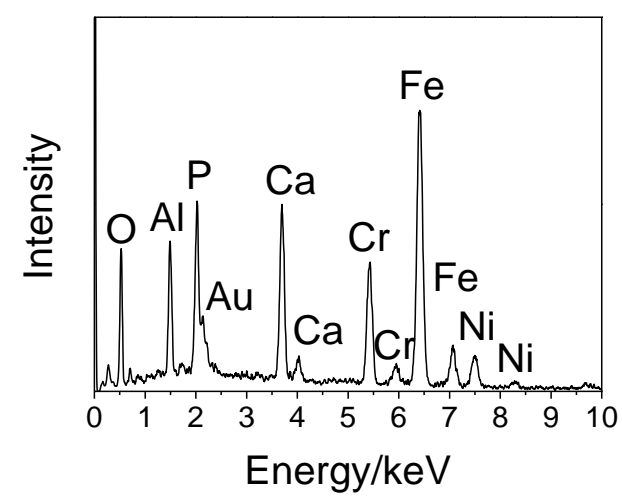

FIGURE 9. EDX result of the surface of the SUS316L substrate after the sandblasting process using the $14 \mu \mathrm{m}$ grinding particles, the AN treatment, and then soaking in SBF for 1 day.

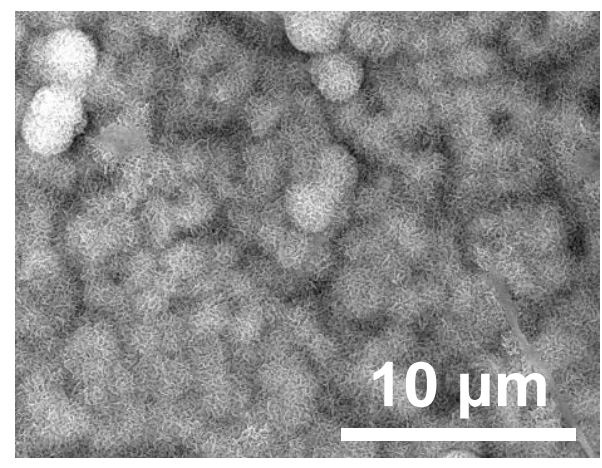

FIGURE 10. SEM picture of the surface of the SUS316L substrate after the sandblasting process using the $3 \mu \mathrm{m}$ grinding particles, the AN treatment, and then soaking in SBF for 1 day.

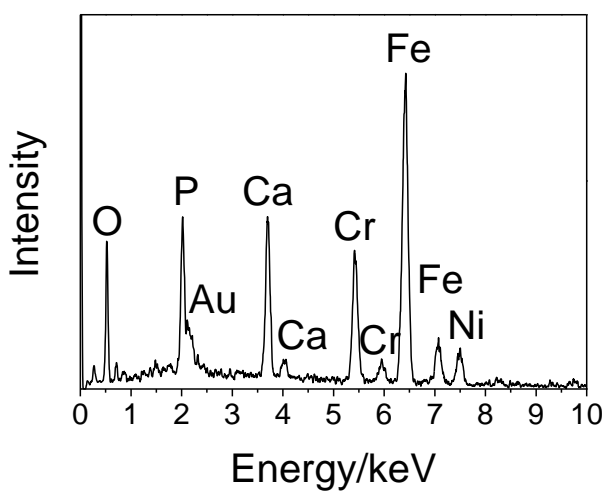

FIGURE 11. EDX result of the surface of the SUS316L substrate after the sandblasting process using the $3 \mu \mathrm{m}$ grinding particles, the AN treatment, and then soaking in SBF for 1 day.

FIGURE 12 shows the average adhesive strengths between the apatite layer formed by soaking in SBF for 14 days and the substrate for the sandblasting process using the $14 \mu \mathrm{m}$ or the $3 \mu \mathrm{m}$ alumina grinding particles. The average adhesive strengths between the formed apatite layer and the specimens were $4.5 \pm 1.3 \mathrm{MPa}$ for $14 \mu \mathrm{m}$ grinding particles and $6.8 \pm 0.9 \mathrm{MPa}$ for $3 \mu \mathrm{m}$ ones, respectively. It is suggested that the smaller grinding particles effectively achieved a mechanical interlocking effect between the apatite layer and the substrate because they showed larger surface roughness in the sandblasting process as shown in FIGURE 4 and FIGURE 5. As a result, higher adhesive strength of the apatite layer was effectively enhanced in comparison with the case of the larger grinding particles. This means that the size of grinding particles in the sandblasting process is an important factor to enhance the adhesive strength of the apatite layer. 


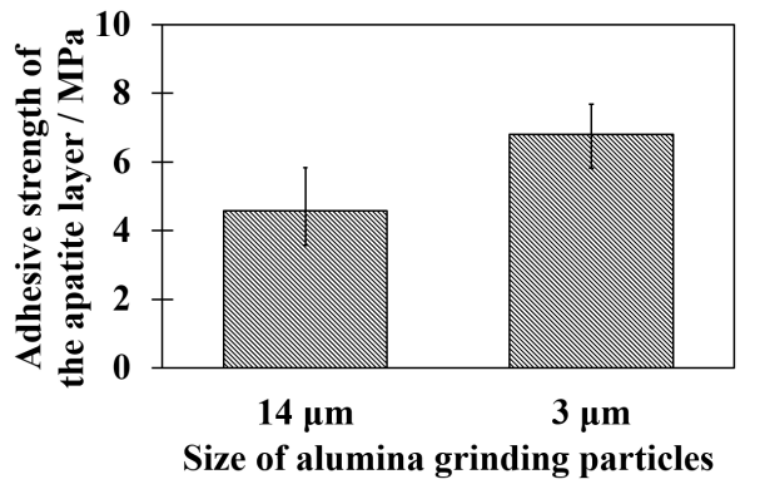

FIGURE 12. Average adhesive strength between the apatite layer formed by soaking in SBF for 14 days and the substrate for the sandblasting process using the $14 \mu \mathrm{m}$ or the $3 \mu \mathrm{m}$ alumina grinding particles.

\section{CONCLUSION}

We formed many micropores on the surfaces of SUS316L substrates by sandblasting methods using two kinds of grinding particles. We provided the apatite-forming ability to the SUS substrates by the AN treatment. From the results of the SBF test, it was indicated that bone-like apatite covered the whole surface of the substrate within 1 day by soaking in SBF regardless of the sandblasting conditions. The bone-like apatite layer showed high adhesive strength to the SUS substrate by a mechanical interlocking effect. From the viewpoint of the surface roughness of the substrate, it is suggested that smaller grinding particles were available to enhance the mechanical interlocking effect and the adhesive strength of the formed apatite layer.

\section{REFERENCES}

1. T. Kokubo, H. Kushitani, S. Sakka, T. Kitsugi, T. Yamamuro, J. Biomed. Mater. Res., 24, 721 (1990)

2. T. Kokubo, H. Takadama, Biomaterials, 27, 2907 (2006).
3. ISO/FDIS 23317, International Organization for Standardization (2007).

4. H. Takadama, T. Kokubo, in Bioceramics and their clinical applications, Edited by T. Kokubo (Woodhead Publishing, Cambridge, 2008), Chap. 7, pp. 165-182.

5. T. Yao, M. Hibino, S. Yamaguchi, H. Okada, U.S. Patent 8,178,066 (2012), Japanese Patent 5,261,712 (2013).

6. T. Yao, M. Hibino, T. Yabutsuka, U.S. Patent 8,512,732 (2013), Japanese Patent 5,252,399 (2013).

7. T. Yao, T. Yabutsuka, PCT Patent PCT/JP2012/76738 (2012).

8. T. Yabutsuka, T. Yao, Key Eng. Mater., 493-494, 545 (2012).

9. H. Mizuno, T. Yabutsuka, T. Yao, Key Eng. Mater., 529-530, 553 (2013).

10. T. Yabutsuka, H. Mizuno, T. Yao, Key Eng. Mater, 587, 165 (2014).

11. T. Yabutsuka, H. Mizuno, R. Karashima, T. Yao, Key Eng. Mater., 631, 231 (2015).

12. T. Shimizu, S. Fujibayashi, B. Ootsuki, M. Takemoto, T. Kiduki, S. Yamaguchi, T. Matsushita, T. Kokubo, S. Mastuda, Abstracts of The 35th Annual Meeting of the Research Society for Orthopaedic Biomaterials, p. 35 (2015).

13. Designation C-633, in Annual Book of ASTM Standards, Vol. 3.01 (American Society for Testing and Materials, 1993), pp. 665-669.

14. W. Lacefield, in An Introduction to Bioceramics, Edited by L. L. Hench, J. Wilson (World Scientific, Singapore, 1993), Chap. 12, pp. 223-238.

\section{ACKNOWLEDGEMENTS}

The authors thank Prof. T. Hoshide (Kyoto University) and Prof. S. Imatani (Kyoto University) for their cooperation on the surface roughness measurements by laser microscopic observation. 